\title{
Touchscreens for All? Angeleitetes Spielen als Einstieg für Senioren und Seniorinnen in digitale Technologien
}

\author{
Sabrina Oppl
}

Eingegangen: 20. November 2019 / Angenommen: 30. Januar 2020 / Online publiziert: 11. Februar 2020 (C) Der/die Autor(en) 2020

Zusammenfassung Durch die fortschreitende Digitalisierung ist für die Bewältigung von vielen Alltagssituationen zunehmend die Nutzung von Touchscreen-basierten Geräten notwendig. Dies kann besonders für Menschen im hohen Erwachsenenalter zu neuen Herausforderungen führen und ihre gesellschaftliche Teilhabe erschweren. Digitale Unterhaltungsspiele am Tablet können beitragen, positive Einstiegserfahrungen mit interaktiven digitalen Medien zu sammeln und die weitere Auseinandersetzung mit diesen Medien fördern. Im vorliegenden Beitrag steht die effektive Unterstützung von Senioren und Seniorinnen bei den ersten spielerischen Auseinandersetzungen mit dem Tablet im Mittelpunkt. Es werden wesentliche Elemente eines praxiserprobten Unterstützungskonzepts eingeführt und ein Leitfaden für Lernbegleiter/innen vorgestellt. Der Leitfaden gibt detaillierte Anleitungen wie ein erfahrungsbasiertes Lehr-Lernarrangement mit vorausgewählten Spielen und einem Scaffolding-Ansatz vorbereitet und umgesetzt werden kann. Die Wirksamkeit des Lehr-Lernarrangements im Sinne der Zielsetzung konnte in mehreren qualitativen Studien gezeigt werden. Der Artikel gibt anhand von Beispielen aus diesen Studien vertieften Einblick in die einzelnen Phasen des Leitfadens, dessen Potentiale und Grenzen der Einsetzbarkeit. Somit finden Praktiker und Praktikerinnen eine Anleitung für die wirksame Unterstützung von älteren Menschen bei den ersten spielerischen Auseinandersetzungen mit Touchscreen-Geräten.

Schlüsselwörter Ältere Menschen · Spielen · Touchscreen · Tablet · Scaffolding · Erfahrungsbasiertes Lernen

\footnotetext{
S. Oppl $(\bowtie)$

Institut für Wirtschaftsinformatik - Communications Engineering, Johannes Kepler Universität Linz, Linz, Österreich

E-Mail: sabrina.oppl@jku.at
} 


\title{
Touchscreens for All? Guiding Elderly People in Their Initial Playful Engagement with Digital Technologies
}

\begin{abstract}
Due to the advancing digitalization, the use of touchscreen-based devices is increasingly necessary in everyday life. This can lead to new challenges, especially for elderly people when aiming to participate in ongoing socio-technological developments. Playing digital entertainment games on a tablet can help to make first positive experiences with interactive digital media and promote their further use. This article deals with adequate support in this game-based learning setting. It describes the different elements of the developed support concept and presents a guide for facilitators. The guide gives detailed instructions on how to prepare and implement an experiential learning setting with preselected games and a scaffolding approach. The effectiveness of the learning setting in terms of the objectives has been demonstrated in several qualitative studies. Using examples from these studies, the article shows the different phases of the guide, its potential and limits of applicability. It offers practitioners guidance on how to successfully support elderly people in their first playful experiences with tablets.
\end{abstract}

Keywords Elderly people · Gaming · Touchscreen · Tablet · Scaffolding · Experiential learning

\section{Spielen als erfolgreicher Einstieg, aber wie?}

Um aktiv an der Informations- und Technologiegesellschaft teilhaben zu können, ist die Nutzung von verschiedenen interaktiven digitalen Medien zunehmend erforderlich (Süss et al. 2018). Gerade im hohen Erwachsenenalter stehen Menschen durch die zunehmende Digitalisierung der Alltagswelt vor neuen Herausforderungen. So sind viele Dienstleistungen, wie etwa Fahrkartenverkauf, nur noch durch die Bedienung von digitalen, mehrheitlich auf Touchscreens basierenden Geräten möglich. Auch wenn immer mehr ältere Menschen neue digitale Medien verwenden (Seifert und Schelling 2016), ist die Gruppe der älteren Bevölkerung bzgl. ihrer Vorerfahrungen sehr heterogen (Stone et al. 2017). Es gibt nach wie vor noch vergleichsweise viele Personen, die mit aktuellen digitalen Medien zu wenig vertraut sind und Schwierigkeiten bei deren Nutzung haben (Barnard et al. 2013). So zeigen Untersuchungen aus unterschiedlichen europäischen Ländern, dass in etwa nur $55 \%$ der Personen, die 65 Jahre oder älter sind, das Internet nutzen (Seifert und Schelling 2016; Statistik Austria 2018.; Statistisches Bundesamt 2017), während der Anteil der Internetnutzer/innen in der Gesamtbevölkerung bei $80 \%$ liegt (Eurostat 2016). Auf Basis dieser Sachverhalte stellt sich die Frage, wie das Erlernen der Nutzung neuer digitaler Technologien für den älteren Teil der Bevölkerung adäquat unterstützt werden kann.

Tippelt und Setzer (2013) geben an, dass bei älteren Menschen häufig ein ,negativ gefärbtes Altersbild“ vorhanden ist, wonach in der Selbstwahrnehmung ,das eigene Lebensalter als zentrale Teilnahmebarriere“ an Weiterbildungsmaßnahmen verstanden wird. Auch kann , die mangelnde Passung zwischen den eigenen Erwartungen 
und den konkreten Angeboten der Bildung eine Teilnahme verhindern" (Tippelt und Setzer 2013). Darüber hinaus stellt das Erlernen des Umgangs mit neuen digitalen Geräten oftmals eine besondere Herausforderung für ältere Menschen dar, da diese nicht mit vergleichbaren digitalen Technologien aufgewachsen sind (Wortley et al. 2017; Chiu und Liu 2017). Die Angst zu scheitern oder etwas falsch zu machen sind zusätzliche Hindernisse im Lernprozess (Oppl und Stary 2018). Ziel sollte es deshalb sein, die ersten Erfahrungen mit interaktiven digitalen Geräten derart positiv erlebbar zu machen, sodass die Bereitschaft bei älteren Menschen entsteht, sich fortgesetzt und mit Freude mit digitalen Technologien auseinanderzusetzen.

Generell ist der Einsatz von Spielen im Bildungsbereich zur Förderung von positiven Lernerfahrungen in vielen verschiedenen Kontexten anzutreffen, wie zahlreiche wissenschaftliche Arbeiten zeigen (Connolly et al. 2012). Durch spielbasiertes Lernen kann eine motivierende Lernumgebung geschaffen werden, um so Wissen und Kompetenzen zu erwerben (ebd). Wissenschaftliche Arbeiten zeigen auch, dass bei älteren Menschen grundsätzlich von einer Offenheit gegenüber digitalen Spielen ausgegangen werden kann (Wang et al. 2011) und dass computerspielende Personen eine Verbesserung der digitalen Kompetenzen durch das Spielen erfahren (Wang et al. 2011; Kaufman et al. 2014).

Untersuchungen weisen darauf hin, dass das Spielen von Unterhaltungsspielen (z. B. Puzzle) am Tablet erste positive Erfahrungen mit interaktiven digitalen Geräten ermöglichen (Springett et al. 2018; Oppl und Stary 2018) und die weitere Auseinandersetzung mit Tablets, Smartphones, oder Fahrkartenautomaten fördern. Es hat sich in diesem Kontext jedoch auch gezeigt, dass der alleinige Einsatz von digitalen Spielen nicht ausreicht, um erfolgreiche Lernerfahrungen zu erzielen. Vielmehr ist individuell angepasste Unterstützung notwendig, um die Herausforderungen der Tabletinteraktion mittels Touchscreen beim Spielen erfolgreich bewältigen zu können (Oppl und Stary 2018). Die Frage der Unterstützung bei der ersten spielerischen Auseinandersetzung mit Tablets wurde im Rahmen des Erasmus+ KA2 Projekts GIRDA (Gameplay for Inspiring Digital Adoption) untersucht. Der vorliegende Artikel berichtet über das entwickelte Unterstützungskonzept und behandelt folgende Fragestellung:

Wie kann ein Unterstützungsangebot ausgestaltet sein, welches erste erfolgreiche Erfahrungen mit interaktiven digitalen Geräten durch Spielen am Tablet bei älteren Menschen fördert?

Zur Beantwortung dieser Frage wurden empirische Erkenntnisse aus einer eigenen Vorstudie (Oppl und Stary 2018) zur Unterstützung erfolgreicher Lernerfahrungen bzgl. der relevanten Einflussfaktoren analysiert. Im Sinne des gestaltungsorientierten Forschungsvorgehens (Peffers et al. 2007) wurden weitere Literaturanalysen durchgeführt, um geeignete Unterstützungskonzepte zu finden und auszuwählen. Dies führte zur Konzeption eines Leitfadens für Lernbegleiter/innen (LB) zur Unterstützung von älteren Menschen bei ihren ersten spielerischen Erfahrungen mit Touchscreens bzw. Tablets. Abb. 1 gibt einen Überblick über den Forschungsprozess zur Entwicklung und Evaluierung des Leitfadens und ermöglicht eine Einordnung der in dieser Arbeit beschriebenen Abläufe. 


\section{Forschungsprozess zur Entwicklung und Evaluierung des Leitfadens}

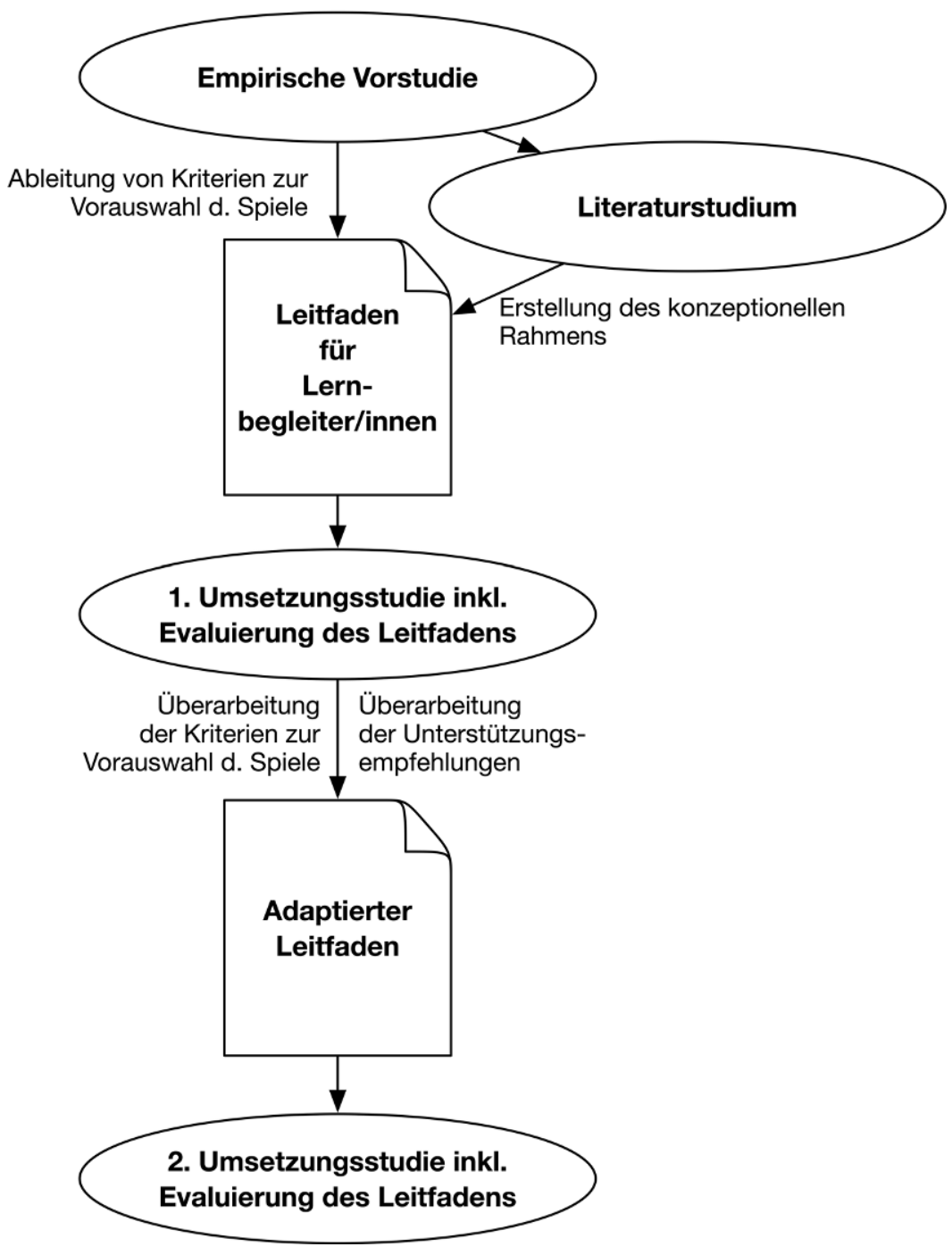

Abb. 1 Forschungsprozess

Der Leitfaden gibt eine detaillierte Anleitung zur Durchführung eines Lehr-Lernarrangements, in welchem ältere Menschen beim Spielen am Tablet eine individualisierte und an den Lernfortschritt angepasste Unterstützung erhalten. Der Leitfaden ist für all jene Menschen konzipiert, welche ältere Menschen in Kleingruppen (oder auch in Einzelbetreuung) bei den ersten Erfahrungen mit interaktiven digita- 
len Geräten unterstützen und somit die Rolle eines/einer LB einnehmen möchten. In diesem Artikel wird zuerst der konzeptionelle Rahmen des Leitfadens beschrieben, bevor die Spielvorauswahl als notwendige Vorbereitungsarbeit des/des LB erläutert wird. Darauffolgend werden die einzelnen Phasen des Leitfadens an Hand praktischer Umsetzungsbeispiele vorgestellt. Abschließend werden die Potentiale und die Grenzen der Einsatzbarkeit des Leitfadens basierend auf den Erkenntnissen von Untersuchungen zur Umsetzung diskutiert. Der Artikel bietet damit Praktikern und Praktikerinnen eine Anleitung zur erfolgreichen Unterstützung älterer Menschen bei den ersten spielerischen Erfahrungen mit interaktiven digitalen Geräten.

\section{Konzeptioneller Rahmen}

Wie in der Einleitung beschrieben, stellten die Erkenntnisse der eigenen Vorstudie (Oppl und Stary 2018) den Ausgangspunkt für die weitere Forschungsarbeit dar. In dieser explorativen Studie machten Menschen im Alter zwischen 57 und 87 Jahre erste Erfahrungen mit Tablets durch das Spielen von Unterhaltungsspielen. Ziel der darauffolgenden Literaturrecherche war es, Theorien und Methoden zu identifizieren, welche den konzeptionellen Rahmen für eine adäquate Unterstützung in einem auf Spiele basierenden Lehr-Lernarrangement bilden können. Dieser Abschnitt gibt einen Überblick über die literaturbasierten Komponenten des Unterstützungskonzepts.

Unterschiedliche wissenschaftliche Arbeiten konzeptualisieren einen auf Spielen basierten Lernprozess durch einen erfahrungsbasierten Lernansatz (z. B. van der Meij et al. 2013). Erfahrungsbasiertes Lernen (Experiential Learning) geht auf Kolb (1984) zurück, welcher einen in vier Phasen gegliederten Lernprozess beschreibt: Eigene Erfahrungen (,concrete experience“) stellen dabei die Basis des Lernens dar. Im vorliegenden Anwendungsfall entsprechen die aktiven Spielerfahrungen am Tablet der ersten Phase nach Kolb. Die Spielerfahrungen alleine würde nach Kolb jedoch zu unzureichenden Lernergebnissen führen. Eine Reflexion (,,reflective observation“) der Erfahrung und daraus abgeleitete abstrakte Konzepte (,,abstract conceptualization“) sollen aktive weitere Auseinandersetzungen (,active experimentation“) mit dem Lerngegenstand - in diesem Fall mit einem interaktiven digitalen Medium - ermöglichen.

Die Daten aus der eigenen Vorstudie deuten jedoch darauf hin, dass eine individualisierte und dynamisch angepasste Unterstützung durch einen/eine LB notwendig ist, um einen erfolgreichen auf digitale Spiele basierten Lernprozess bei älteren Personen ohne digitale Vorerfahrungen zu fördern (Oppl und Stary 2018). Scaffolding (van de Pol et al. 2010) ermöglicht eine derartige Unterstützung, durch eine an den Bedürfnissen der Lernenden angepasste Hilfestellung. Dieses Konzept sieht eine laufende Beobachtung der Lernenden und eine dynamische Anpassung der Unterstützungsmaßnahmen im Zeitverlauf vor, um den Lernenden die Kontrolle über deren Handlungen soweit wie möglich zu übertragen. Dabei ist es entscheidend, dass die Lernenden möglichst selbstständig arbeiten und nur dann Hilfestellungen bekommen, wenn sie die nächsten Schritte nicht alleine erreichen können. Die individuellen Erfolge oder Schwierigkeiten sollen immer den Bezugspunkt für die 
folgenden Instruktionen darstellen. Die Anwendung von Scaffolding beim Spielen am Tablet bedeutet somit die Förderung von weitgehend eigenständiger Spielphasen, wobei genau jene Hilfestellungen gegeben werden sollen, die notwendig sind, um erfolgreiche neue Lernerfahrungen zu fördern. Je nach Bedarf kann der/die LB mit Erklärungen, Hinweisen, Fragen oder Demonstrationen am Tablet unterstützen. Die Art und Intensität ist von dem/der LB situationsgerecht und dynamisch zu entscheiden. Eine an den Prinzipien von Scaffolding orientierte Hilfestellung kann in diesen erfahrungsbasierten Lernansatz integriert werden, da in beiden Konzepten das selbstständige Handeln der Lernenden die Basis des Lernprozesses darstellt.

Hilfestellungen sind jedoch nicht nur im Spielprozess notwendig. Auch die von Kolb beschriebene Reflexion und die Ableitung von generalisierbaren Schlussfolgerungen bedarf gezielter Unterstützung, da es für Spieler/innen oft schwierig ist, ihre Spielerfahrung auf die Herausforderungen der realen Welt abzubilden (Rotter et al. 1981). Mehrere Studien beschreiben Debriefing-Methoden als geeignet zur Reflexion von Spielerfahrungen. An das Spielen und den Lernzyklus von Kolb angepasste Debriefing-Fragen können den Reflexions- und Abstraktionsprozess unterstützen (z. B. Walther 2013). Für den vorliegenden Anwendungsfall bedeutet dies somit eine Anpassung der in der Literatur vorgeschlagenen Debriefing-Fragen als Hilfestellung für den/die LB, um den Reflexions- und Abstraktionsprozess zu bei den Lernenden zu unterstützen. Die Fragen können dementsprechend wie folgt lauten: Was ist Ihnen gelungen? - Warum? Welche Probleme sind aufgetreten? - Warum? Wie sind Sie vorgegangen um ...? Glauben Sie, dass Sie diese Erfahrungen in anderen Situationen mit einem ähnlichen Gerät (Tablet, Smartphone, Fahrkartenautomat) brauchen können? Wenn ja, warum?

Das Unterstützungskonzept orientiert sich somit an den von Kolb beschriebenen Phasen des auf Erfahrung basierenden Lernens. Während die erstgenannte Phase die aktive Auseinandersetzung mit dem Lerngegenstand (Spielen am Tablet) abbildet, beschreiben die zweite und dritte Phase die Reflexion und die davon abgeleiteten Schlussfolgerungen der zuvor gewonnenen Lernerfahrungen, um diese in neuen Situationen anzuwenden bzw. mit diesen zu experimentieren (durch erneutes Spielen oder anderwärtige Auseinandersetzung mit dem Tablet). Durch die Anwendung von Scaffolding und Debriefing sollen die Lernenden durch den/die LB jene Unterstützung erhalten, welche notwendig ist, um den erfahrungsbasierten Lernprozess erfolgreich bewältigen zu können. Um im Sinne von Scaffolding erfolgreiche Lernerfahrungen zu fördern, zeigt der folgende Abschnitt die Notwendigkeit einer angepassten Vorauswahl an Spielen auf.

\section{Vorauswahl der Spiele}

Das im letzten Abschnitt beschriebene Scaffolding-Konzept sieht eine Anpassung der Unterstützung an die Bedürfnisse der Lernenden vor. Dies impliziert auch die Auswahl der Lernaufgaben, um Über- und Unterforderungen zu vermeiden (van de Pol et al. 2010). Demzufolge müssen auch die Spiele den Voraussetzungen der Lernenden entsprechend ausgewählt werden. Die im Folgenden dargestellten Kriterien der Spielauswahl basieren auf den Erkenntnissen der Vorstudie und der ersten Um- 
setzungsstudie zum vorliegenden Unterstützungskonzept (siehe Abb. 1 Forschungsprozess).

Die Datenanalyse der Vorstudie führt zur Empfehlung, thematisch verschiedenartige Spiele auszuwählen, um die unterschiedlichen Interessen der Lernenden zu berücksichtigen. Die gewählten Spielen sollen in der realen - nicht digitalen - Welt existieren und den Lernenden bekannt sein, um die initiale Komplexität der Gerätehandhabung durch möglichst vertraute Spiele nicht noch weiter zu erhöhen. Derartige Spiele sind beispielsweise Puzzles, Memory, Kreuzworträtsel oder Sudoku. Auch wenn diese digitalen Spiele alle ein Pendant in der realen Welt haben, unterscheiden sie sich im Ausmaß der realitätsgetreuen Abbildung. So sind bei den meisten Puzzle-Spielen für Tablets die Puzzleteile durch Antippen und Schieben zusammenzuführen. Dies ist ein dem realen Puzzle-Spiel ähnlicher Handlungsablauf und ist - wie sich in den Untersuchungen zeigte - von den Senioren/innen leicht nachvollziehbar. Anders ist dies beispielsweise bei einer Kreuzworträtsel-App, da hier üblicherweise die Eingabe der Wörter durch eine Tastatur am Bildschirm erfolgt, wodurch sich einerseits die Anforderungen an die Bedienung des Tablets erhöhen und anderseits weniger Parallelen zu einem Kreuzworträtsel in einer Tageszeitung gezogen werden können. Für die ersten Versuche bei der Bedienung eines Touchscreen-basiertes Geräts empfiehlt der Leitfaden eine Auswahl an Spielen, die nicht nur in der realen Welt existieren, sondern auch möglichst realitätsnah zu bedienen sind, um einen möglichst niederschwelligen Einstieg zu ermöglichen. Haben die Lernenden hingegen bereits erste Erfahrungen mit derartigen Geräten gesammelt bzw. werden mehrere Spielrunden absolviert, können auch Spiele mit weniger realitätsgetreuer Bedienung verwendet werden. Die erste Umsetzungsstudie führte zu weiteren Hinweisen für die Vorauswahl und zu dementsprechenden Ergänzungen bei den Empfehlungen: Bei den Spielen ist darauf zu achten, dass keine schnellen Handlungsreaktionen notwendig und keine Zeitlimits vorgesehen sind. Beim Einsatz eines Kartenspiels, in dem die Spielprinzipien hohe Reaktionsgeschwindigkeit belohnen, zeigte sich zum Beispiel, dass dieses von den Teilnehmer/innen auf Grund dessen Bekanntheit zwar gerne gewählt wurde, aber Zeitlimits in den einzelnen Spielrunden zu Stress und ausbleibenden Erfolgen führten. Darüber hinaus zeigte die Untersuchung, dass die einzelnen Spielrunden nicht zu lange sein sollten (max. $15 \mathrm{~min}$ ), um rasch Erfolge zu erzielen und Ermüdungseffekte zu vermeiden. Bei Puzzlespielen sollten daher eher Puzzles geringer Komplexität verwendet werden (z.B. weniger als 20 Teile, leicht erkennbare Muster).

Die nach den oben beschriebenen Kriterien ausgewählten Spiele sollen am Tablet installiert und als Übersicht auf Papier (Titel der Spiele inkl. Kurzbeschreibungen) zur Auswahl vorbereitet werden. Diese Vorbereitungen stellen die Grundlage für ein erfolgreiches Scaffolding dar und sind somit als eine wesentliche Komponente des vorliegenden Unterstützungskonzepts anzusehen.

\section{Aufbau und Umsetzung des Leitfadens}

Um eine Umsetzung des in dieser Arbeit dargestellten Unterstützungskonzepts zu erleichtern, wurde ein Leitfaden für LB mit detaillierten Anleitungen und Empfeh- 
lungen erstellt. Abb. 1 gibt einen Überblick über die Phasen des Leitfadens, bevor dessen praktische Umsetzung mit beispielhaften Ausführungen erläutert wird. Die Beispiele stammen aus zwei Evaluierungen des Leitfadens mit insgesamt 33 Senior/ innen, die im Rahmen des Erasmus+ KA2 Projekts GIRDA (www.girda.eu) durchgeführt wurden. In einem quasi-experimentellen Untersuchungssetting wurden Videoaufzeichnungen erstellt und Interviews mit allen Teilnehmer/innen durchgeführt. Die Umsetzung erfolgte - so wie im Leitfaden vorgesehen - meist jeweils mit zwei Teilnehmer/innen. Die Rolle des/der LB wurde von Studenten und Studentinnen der Wirtschaftsinformatik der JKU übernommen, welche ohne spezielle Schulungsmaßnahmen oder pädagogische Vorerfahrungen die Lehr-Lernarrangements ausschließlich auf Basis des Leitfadens vorbereiteten und durchführten.

Spielauswahl durch die Lernenden Die Teilnehmer/innen wurden empfangen und über den Ablauf informiert. Den Teilnehmer/innen ohne jegliche Vorerfahrung wurde die Liste mit Spielen mit realitätsnaher Bedienung vorgelegt und sie wurden zur freien Spielauswahl ermutigt. Teilnehmer/en mit ersten Vorerfahrungen wurde die zweite Liste vorgelegt. Die Analyse der Interviewdaten zeigt, dass die Möglichkeit ein Spiel frei auszuwählen insgesamt positiv bewertet wurde. Meist wurde das Spiel interessensbezogen ausgewählt, wobei auch die persönliche Einschätzung, dass ein Spiel „einfacher“ (i.S.v. weniger komplexer Spielmechanik) sei als die anderen, als Entscheidungskriterium genannt wurde.

Spielen am Tablet Nach der Auswahl eines Spiels wurde den Teilnehmer/innen jeweils ein Tablet mit den vorinstallierten Spielen vorgelegt. Der/die LB öffnete das Spiel und machte einführende Erklärungen mit kurzen Demonstrationen. So wurde beispielsweise beim Puzzle vorgezeigt, wie die Puzzleteile bewegt werden können. Nach der ersten kurzen Einführung wurden die Teilnehmer/innen ermutigt, das Spiel selbst auszuprobieren. Hier kam es üblicherweise zu ersten Rückfragen oder Schwierigkeiten. Bei einer Teilnehmerin mit dem Spiel „Malen nach Zahlen“ musste beispielsweise das Vergrößern und Verkleinern des Bildschirms ein zweites Mal vorgezeigt werden, um die einzelnen Felder einfärben zu können. Bei der Verwendung von kostenfreien Spielen wurden zum Teil zwischendurch Werbefenster eingeblendet, was zu Verunsicherungen bei den Teilnehmer/innen führte. Der/die LB gab daraufhin Erklärungen und Anleitungen, wie ein Werbefenster wieder geschlossen werden kann. Zu Problemen und somit zu Hilfestellungen kam es darüber hinaus vorwiegend beim Start neuer Spielrunden und bei abweichenden Handlungen bzw. Ereignissen (z.B. ein anderes Fenster öffnete sich auf Grund unabsichtlicher fehlerhafter Bedienung). Das Spielen selbst war nach einer ersten Einführung überwiegend eigenständig möglich und erfolgreich. Abhängig von der Spieldauer kam es nach ein bis drei Spielrunden zu einer Beendigung der Spielephase durch den/die LB und es wurde zur nächsten Phase übergeleitet.

Reflektieren \& Abstrahieren Der/die LB führte auf Basis der Debriefing-Fragen (z.B. Was ist Ihnen beim Spielen gelungen/nicht gelungen? Wie sind Sie vorgegangen um ...?) mit jedem/jeder Teilnehmer/in ein kurzes Gespräch zu den Spielerfahrungen, während der/die andere Teilnehmer/in weiterspielte oder beim Gespräch 
zuhörte. Die Teilnehmer/innen wurden gebeten, die erfolgreichen Spielerfahrungen und auch die diesbezüglichen konkret gesetzten Handlungen zu beschrieben. Danach wurde mit den Teilnehmer/innen darüber gesprochen, wo und wie diese Lernerfahrungen unter Umständen in anderen Situationen mit vergleichbaren digitalen Geräten angewendet werden können, um den Transfer des Gelernten zu unterstützen (z.B. Werbefenster erscheinen etwa nicht nur bei Spielen, sondern häufig auch bei der Nutzung von Websites im Browser $\rightarrow$ Nachbesprechung, wie diese unerwünschten Fenster geschlossen werden können). Bei den weniger erfolgreichen Spielerfahrungen bzw. bei den aufgetretenen Problemen wurde gleich vorgegangen. In dieser Phase zeigte sich, dass es Teilnehmer/innen schwerfallen kann, über die Details der erfolgreichen Handlungsabläufe zu sprechen, während über die Probleme meist sehr ausführlich berichtet werden konnte. Durch gezieltes Nachfragen bzw. durch beispielhafte Ausführungen durch den/die LB wurde versucht, auch die von den Teilnehmer/innen nicht angesprochenen Erfahrungen aufgrund der Beobachtungen zu erfassen. So beschrieb beispielsweise der/die LB die einzelnen - von einer Teilnehmerin gesetzten - Handlungen, die zur erfolgreichen Bewegung von Puzzleteile geführt haben, um nicht nur die Probleme, sondern auch die erfolgreichen Handlungen explizit zu reflektieren.

Experimentieren am Tablet Nach der Nachbesprechung der Spielerfahrungen konnten die Teilnehmer/innen ihre weiteren Aktivitäten am Tablet frei wählen. Es wurde die Möglichkeit aufgezeigt, das zuvor gespielte Spiel erneut zu spielen, ein neues Spiel (auch aus der anderen Liste) zu wählen, oder das Tablet (ohne den Einsatz von Spielen) zu erkunden. Die Entscheidungen fielen sehr unterschiedlich aus. Während beispielsweise ein Teilnehmer das zuvor begonnene Kreuzworträtsel weiter fortsetzen wollte, erprobte eine Teilnehmerin das Fotografieren mit dem Tablet, während eine andere eine Nachrichtenseite im Internet zu bedienen übte. Das Ausmaß der in dieser Phase angebotenen Hilfestellungen war sehr unterschiedlich, je nach Teilnehmer/innen und gewünschten Anwendungen.

Erprobt wurde der Leitfaden im Rahmen der Evaluierungen mit einem einmaligen Durchlauf der einzelnen Phasen. Der hinaufführende Pfeil in Abb. 2 zeigt hingegen, dass dies mehrmals möglich ist bzw. dass die Phase des Experimentierens durch die Auswahl eines neuen Spieles fließend in einen neuen Durchlauf übergehen kann. Nach der beispielhaften Beschreibung der Umsetzung der einzelnen Phasen, zeigt der folgende Abschnitt nun die Potentiale und Grenzen der Einsetzbarkeit des Leitfadens auf.

\section{Potentiale und Grenzen der Einsatzbarkeit}

In diesem Beitrag wurde gezeigt, wie Senior/innen bei den ersten Erfahrungen am Tablet mit vorausgewählten Unterhaltungsspielen durch die Anwendung von Scaffolding und Debriefing unterstützt werden können. Es wurde ein Leitfaden vorgestellt, der die Umsetzung des konzipierten Unterstützungskonzepts für LB erleichtern soll. In diesem abschließenden Abschnitt werden unterschiedliche Aspekte der Einsatzbarkeit des Leitfadens diskutiert. 


\section{Leitfaden}

\section{zur Unterstützung von ersten spielerischen Erfahrungen mit Tablets}

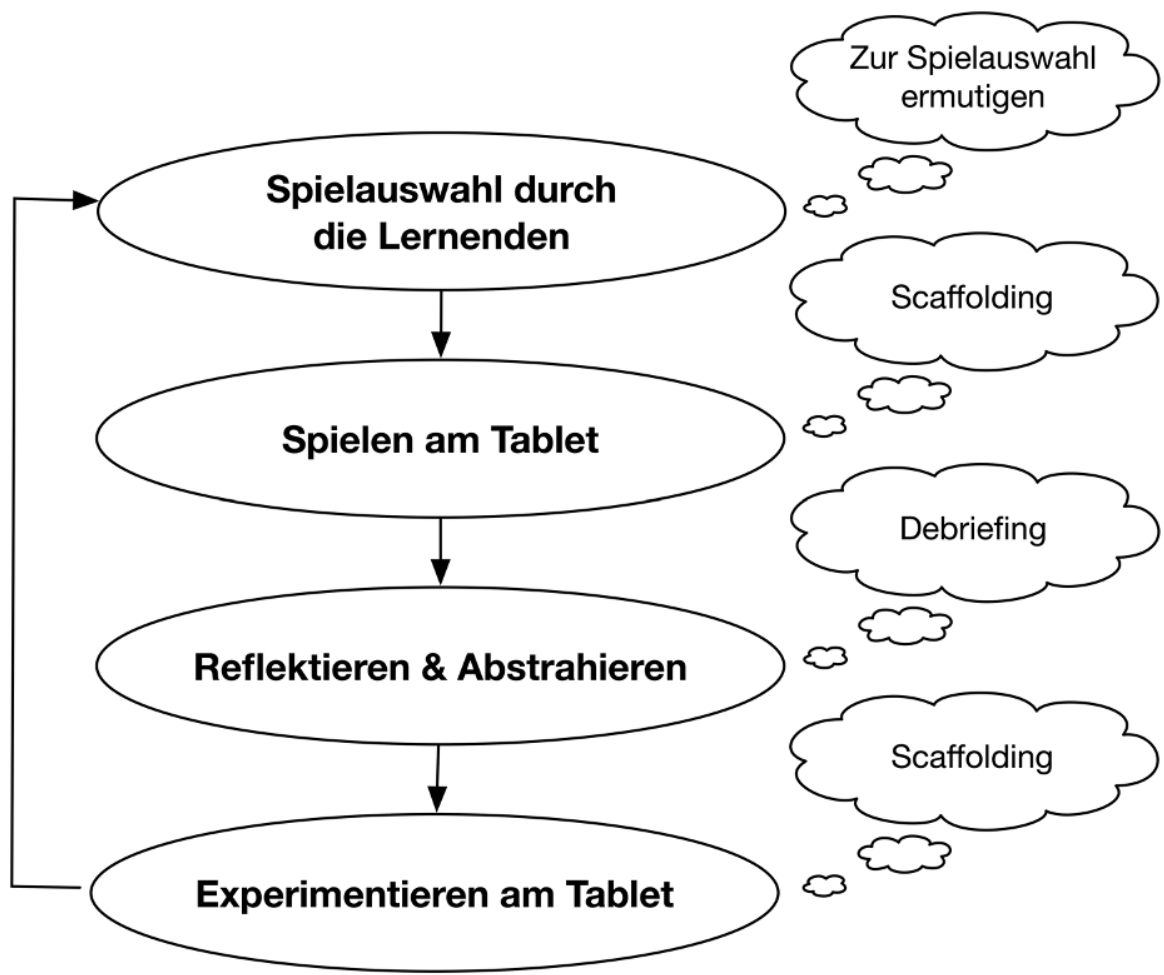

Abb. 2 Leitfaden für Lernbegleiter/innen

Die obigen Darstellungen zur Umsetzung des Leitfadens beziehen sich auf das Vorgehen und die Erkenntnisse aus zwei Studien mit insgesamt 33 Teilnehmer/innen (zwischen 60 und 88 Jahre) und 4 Studenten und Studentinnen der Wirtschaftsinformatik als LB. Es konnte festgestellt werden, dass der Leitfaden von Personen ohne pädagogische Vorerfahrungen oder spezielle Schulungsmaßnahmen umgesetzt werden kann. Die Studenten und Studentinnen erhielten lediglich die Aufgabe, das Lernsetting gemäß den Beschreibungen des Leitfadens vorzubereiten und bei den Evaluierungsterminen umzusetzen. Aus den bisherigen Erfahrungen kann daher angenommen werden, dass der Leitfaden von interessierten Personen mit einer Alltagsmedienkompetenz im Bereich der Tabletnutzung angewendet werden kann. Für eine 
Überprüfung dieser Annahme sind jedoch noch weitere empirische Untersuchungen notwendig.

In diesem Beitrag wurde der Fokus auf die Beschreibung des Einsatzes des Leitfadens bei Senior/innen ohne bzw. mit wenig Vorerfahrungen mit Touchscreen-basierten Geräten gelegt. In einer ergänzten Version des Leitfadens sind auch Szenarien für die Einbindung von Senior/innen mit umfassenderen Vorerfahrungen vorgesehen. In unseren Untersuchungen hat sich gezeigt, dass sich auch diese Gruppe oftmals als Anfänger/innen einschätzt und sich dementsprechend zur Teilnahme gemeldet haben. Die Selbsteinschätzung entspricht somit nicht immer der Fremdeinschätzung. Aus diesem Grund hat es sich als hilfreich herausgestellt, vor der Spielauswahl einige Diagnosefragen einzuführen, um die tatsächlichen Lernvoraussetzungen zu erfragen und um eine optimale Spielauswahl und Unterstützung zu gewährleisten. Für die erfahreneren Teilnehmer/innen sind im ergänzten Leitfaden Spiele vorgesehen, welche keinen Bezug zu in der realen Welt existierenden Spielen haben, um neue Lernerfahrungen mit interaktiven digitalen Medien auf einer höheren Abstraktions- und Komplexitätsstufe zu fördern. Im vorliegenden Beitrag musste aus Platzgründen auf detaillierte Ausführungen zum Diagnoseinstrument und zur erweiterten Spielauswahl verzichtet werden. Die hier dargestellte Variante eignet sich dementsprechend für homogene Gruppen von Anfänger/innen, da die nicht behandelten Elemente vor allem bei einer heterogeneren Zusammensetzung der Teilnehmer/innen relevant sind. Der vollständige Leitfaden ist unter www.girda.eu abrufbar.

In den Untersuchungen hat sich gezeigt, dass das beschriebene Unterstützungskonzept von einem LB bei bis zu zwei Teilnehmer/innen zufriedenstellend umgesetzt werden kann. Ein Erhebungstermin wurde mit drei Teilnehmer/innen und einem LB durchgeführt, was zu einer sehr anspruchsvollen Situation für den LB geführt hat. Auf Basis der Videoanalyse konnte hier von keiner idealen Betreuung ausgegangen werden. Wieweit die Umsetzung des Leitfadens auch bei mehr als zwei Personen möglich ist und zu einer erfolgreichen Auseinandersetzung mit dem Tablet führen kann, hängt vermutlich auch von der Betreuungsintensität der jeweiligen Teilnehmer/ innen ab und bedarf weiterer Untersuchungen.

Auch wenn sich auf Basis dieser Erkenntnisse die Frage nach einer möglichen Umsetzung in einem größeren, beispielsweise öffentlichen Setting stellt, wurde gerade die geringe Anzahl an Teilnehmer/innen pro LB von diesen als besonders positiv bewertet. Im anschließenden Interview wurde häufig die Betreuung durch den/die LB hervorgehoben. Alle Teilnehmer/innen gaben an, sich während der gesamten Teilnahme - trotz Kameraaufzeichnungen - sehr wohlgefühlt zu haben, was meist mit der Anwesenheit, Unterstützung und Aufmerksamkeit des/der LB begründet wurde.

Die Datenanalyse zeigt auch, dass der spielbasierte Ansatz von fast allen Teilnehmer/innen positiv bewertet wurde. Konnte ein Spiel weitgehend eigenständig bewältigt werden, wurde dies als Erfolgserlebnis wahrgenommen. Zentral scheint neben der Unterstützung durch die LB die Vorauswahl der Spiele zu sein. Spiele mit Zeitlimits, raschen Handlungsabläufen oder umfangreichen Konfigurationsmöglichkeiten können zu Überforderungen und zu ausbleibenden Erfolgserlebnissen führen. Ein Teilnehmer hob hervor, die spielerische Auseinandersetzung mit dem Tablet abzulehnen, was er mit einer generellen Abneigung gegenüber Spielen begründete. 
Doch auch er gab positive Rückmeldungen zum Konzept auf Grund der Betreuung. Die insgesamt positiven Äußerungen zum Spielen am Tablet zur Sammlung erster Erfahrungen mit interaktiven digitalen Geräten, entspricht den Erkenntnissen der Literatur, in der von einer Offenheit von älteren Menschen gegenüber digitalen Spielen ausgegangen wird (Wang et al. 2011). Dennoch scheint - basierend auf den vorliegenden Untersuchungen - das Spielen nur ein Teilaspekt unterschiedlicher Unterstützungsmaßnahmen zu sein, um bei den ersten digitalen Spielerfahrungen positive Lernerfahrungen zu fördern.

Danksagung Die Autorin bedankt sich bei Sebastian Gruber, Philip Schuster, Alexander Simon, Rebecca Wernig und Sarah Zeiml für die Unterstützung bei der Durchführung der empirischen Studien und beim Seniorenbund OÖ für die Hilfe bei der Gewinnung der Teilnehmer/innen.

Förderung Die vorliegende Forschung wurde zum Großteil durch das Erasmus+ KA2 Programm (Grant Nr. 2016-1-UK01-KA204-024508) im Rahmen des Projekts GIRDA (Gameplay for Inspiring Digital Adoption) finanziert.

Funding Open access funding provided by Johannes Kepler University Linz.

Open Access Dieser Artikel wird unter der Creative Commons Namensnennung 4.0 International Lizenz veröffentlicht, welche die Nutzung, Vervielfältigung, Bearbeitung, Verbreitung und Wiedergabe in jeglichem Medium und Format erlaubt, sofern Sie den/die ursprünglichen Autor(en) und die Quelle ordnungsgemäß nennen, einen Link zur Creative Commons Lizenz beifügen und angeben, ob Änderungen vorgenommen wurden.

Die in diesem Artikel enthaltenen Bilder und sonstiges Drittmaterial unterliegen ebenfalls der genannten Creative Commons Lizenz, sofern sich aus der Abbildungslegende nichts anderes ergibt. Sofern das betreffende Material nicht unter der genannten Creative Commons Lizenz steht und die betreffende Handlung nicht nach gesetzlichen Vorschriften erlaubt ist, ist für die oben aufgeführten Weiterverwendungen des Materials die Einwilligung des jeweiligen Rechteinhabers einzuholen.

Weitere Details zur Lizenz entnehmen Sie bitte der Lizenzinformation auf http://creativecommons.org/ licenses/by/4.0/deed.de.

\section{Literatur}

Barnard Y, Bradley MD, Hodgson F, Lloyd AD (2013) Learning to use new technologies by older adults: perceived difficulties, experimentation behaviour and usability. Comput Human Behav 29:1715-1724. https://doi.org/10.1016/j.chb.2013.02.006

Chiu C-J, Liu C-W (2017) Understanding older adult's technology adoption and withdrawal for elderly care and education: mixed method analysis from national survey. J Med Internet Res. https://doi.org/ 10.2196/jmir.7401

Connolly TM, Boyle EA, MacArthur E et al (2012) A systematic literature review of empirical evidence on computer games and serious games. Comput Educ 59:661-686. https://doi.org/10.1016/j.compedu. 2012.03.004

Eurostat (2016) Internetnutzung durch Privatpersonen - Nahezu acht von zehn Internetnutzern in der EU surften 2016 mit einem Handy oder Smartphone

Kaufman D, Sauvé L, Renaud L, Duplàa E (2014) Benefits and barriers of older adultsź digital gameplay. In: Proceedings of the 6th international conference on computer supported education, Bd. 1. SCITEPRESS - Science and Technology Publications, Lda, S 213-219

Kolb DA (1984) Experiential learning: experience as the source of learning and development. Prentice Hall, Englewood Cliffs

van der Meij H, Leemkuil H, Li J-L (2013) Does individual or collaborative self-debriefing better enhance learning from games? Comput Human Behav 29:2471-2479. https://doi.org/10.1016/j.chb.2013.06. 001 
Oppl S, Stary C (2018) Game-playing as an effective learning resource for elderly people: encouraging experiential adoption of touchscreen technologies. Univers Access Inf Soc. https://doi.org/10.1007/ s10209-018-0638-0

Peffers K, Tuunanen T, Rothenberger MA, Chatterjee S (2007) A design science research methodology for information systems research. J Manag Inf Syst 24:45-77. https://doi.org/10.2753/MIS07421222240302

van de Pol J, Volman M, Beishuizen J (2010) Scaffolding in teacher-student interaction: a decade of research. Educ Psychol Rev 22:271-296. https://doi.org/10.1007/s10648-010-9127-6

Rotter NG, Stadskleve R, Bredemeier ME (1981) „The academic game“ as a frame game. J Exp Learn Simul 3:73-83

Seifert A, Schelling HR (2016) Alt und offline? Z Gerontol Geriat 49:619-625. https://doi.org/10.1007/ s00391-015-0965-1

Springett M, Keith S, Whitney G (2018) Game-based introductory learning: teaching digital skills to older citizens

Statistik Austria IKT-Einsatz in Haushalten. https://www.statistik.at/web_de/statistiken/energie_umwelt_ innovation_mobilitaet/informationsgesellschaft/ikt-einsatz_in_haushalten/index.html. Zugegriffen: 19. Juni 2018

Statistisches Bundesamt (2017) Staat \& Gesellschaft - IT-Nutzung - Statistisches Bundesamt (Destatis). https://www.destatis.de/DE/ZahlenFakten/GesellschaftStaat/EinkommenKonsum Lebensbedingungen/ITNutzung/ITNutzung.html. Zugegriffen: 19. Juni 2018

Stone ME, Lin J, Dannefer D, Kelley-Moore JA (2017) The continued eclipse of heterogeneity in gerontological research. J Gerontol B Psychol Sci Soc Sci 72:162-167. https://doi.org/10.1093/geronb/ gbv068

Süss D, Lampert C, Trültzsch-Wijnen CW (2018) Medienpädagogik: Ein Studienbuch zur Einführung. Springer, Berlin Heidelberg

Tippelt R, Setzer B (2013) Altersbilder, Bildungsarbeit und Persönlichkeitsentwicklung. In: Weisheit und Wissenschaft: Festschrift zum 25-jährigen Bestehen des Seniorenstudiums an der LMU. Utz, München, S 247

Walther LRFZJ (2013) Debriefing topics and their effects on learning with computer games. https://essay. utwente.n1/64211/. Zugegriffen: 23. Juli 2019

Wang F, Lockee BB, Burton JK (2011) Computer game-based learning: perceptions and experiences of senior Chinese adults. J Educ Technol Syst 40:45-58. https://doi.org/10.2190/ET.40.1.e

Wortley D, An J-Y, Heshmati A (2017) Tackling the challenge of the aging society: detecting and preventing cognitive and physical decline through games and consumer technologies. Healthc Inform Res 23:87-93. https://doi.org/10.4258/hir.2017.23.2.87 